\section{Clinical and microbiological characteristics of community- acquired methicillin-resistant Staphylococcus aureus pneumonia}

\author{
Eric Martin, ${ }^{1}$ Jane Colmer-Hamood, ${ }^{2}$ \\ Wesam Frandah, ${ }^{1}$ Rishi Raj, ${ }^{1}$ \\ Kenneth Nugent ${ }^{1}$
}

Departments of 'Internal Medicine and 2Microbiology, Texas Tech University

Health Sciences Center, Lubbock, TX, USA

\begin{abstract}
Methicillin-resistant Staphylococcus aureus (MRSA) infections now occur in healthy adults in community settings. We searched the PubMed database to identify relevant articles on the clinical presentation, epidemiology, virulence, and treatment of community-acquired MRSA (CA-MRSA) infections, including pneumonia. This information was summarized in a narrative review.MRSA infections cause approximately 30 infections per 100,000 people per year in the USA, and twenty percent of these infections are secondary to CA-MRSA. These community-acquired infections often involve the skin and subcutaneous tissue but can also involve visceral tissues such as the lung and bone. The overall mortality in patients with invasive disease is approximately $10 \%$; it approaches $50 \%$ in patients with pneumonia. The bacterial isolates from these infections have the staphylococcal chromosome cassette mec types 4 and 5 . This genetic characteristic produces beta-lactam resistance and helps distinguish these isolates from hospital-acquired MRSA, which usually have mec types 1-3. Some CA-MRSA isolates release the Panton-Valentine leukocidin (PVL), which causes neutropenia and tissue necrosis; other toxins also contribute to the virulence of these infections. Empiric therapy should include vancomycin or linezolid. CA-MRSA infections can have fulminant courses and high mortality rates. Physicians should consider these infections as possible emergencies with a high risk for organ system failure and shock.
\end{abstract}

\section{Introduction}

Methicillin-resistant Staphylococcus aureus (MRSA) infections first occurred in hospitalized patients or in patients who had previously been hospitalized. However, in the 1990's, MRSA infections began to occur in young, healthy adults in the community. ${ }^{1}$ These latter infections have been called communityacquired MRSA (CA-MRSA) in contrast to hospital-acquired MRSA (HA-MRSA) and are the presumptive diagnosis when the criteria for HA-MRSA are not met. ${ }^{2}$ Studies in these two groups of patients and with the associated bacterial isolates have demonstrated that there are important differences between CA- and HA- MRSA. We recently helped care for a fatal case of CA-MRSA pneumonia, and this stimulated us to review the clinical and microbiological characteristics of CA- and HA-MRSA infections. ${ }^{3}$ We approached this review by answering important clinical questions about these infections to update clinicians who take care of patients with severe infections, especially pneumonia, in intensive care units (ICUs).

\section{Materials and Methods}

PubMed was searched to identify articles on MRSA infections to answer the questions discussed below. We used the following MeSH terms; methicillin-resistant Staphylococcus aureus, pneumonia, virulence factors, community-acquired infection, cross infection, mecA protein $S$. aureus, and epidemiology. MRSA was combined (AND) with the other MeSH terms and the text words Panton-Valentine leukocidin for searches. We used the following limits: published in the last 10 years, English only, and all adults: $19+$ years. Reference lists from pertinent articles and from related articles algorithm provided by PubMed were also reviewed. This information was summarized to create a narrative review.

\section{What is the epidemiology of methicillin-resistant}

Staphylococcus aureus infections in the US? What are the usual clinical

\section{presentations?}

The prevalence of CA-MRSA infections and the typical clinical presentations clearly depend on the population studied. Kuehnert and coworkers determined the prevalence of staphylococcal colonization in a representative sample in the US population using the National Health and Nutrition Examination Survey. ${ }^{4}$ This study involved 9622 subjects and demonstrated that $0.8 \%$ (95\% CI: $0.4-1.4 \%$ ) had nasal colonization with MRSA. This occurred more frequently in women and in participants older than 60 . Fifty percent of the isolates had SCCmec IV genotype, 50\% had the SCCmec II genotype, and $8 \%$ belonged to the USA300 clone (discussed below in the section on genetic studies). The prevalence of MRSA colonization increases in populations with health care associated risk factors, such as chronic dialysis, and some populations with close phys-
Correspondence: Kenneth Nugent, Department of Internal Medicine, Texas Tech University Health Sciences Center, 3601 4th St, Lubbock, TX 79430 USA.

Tel. +1.806.743.3155 - Fax: +1.806 .743 .3148 .

E-mail: kenneth.nugent@ttuhsc.edu

Key words: community-acquired, hospitalacquired, methicillin-resistance, pneumonia, Staphylococcus aureus, Panton-Valentine leukocidin, $\mathrm{SCC}$ mec.

Conflicts of interests: the authors declare that they have no conflicts of interests.

Received for publication: 10 January 2012.

Revision received: 4 March 2012.

Accepted for publication: 6 March 2012.

This work is licensed under a Creative Commons Attribution NonCommercial 3.0 License (CC BYNC 3.0).

(C) Copyright E. Martin et al., 2012

Licensee PAGEPress, Italy

Chest Disease Reports 2012; 2:e7

doi:10.4081/cdr.2012.e7

ical proximity, such as in jails and with some sports activities.

Caffrey and LaPlante reported information on the epidemiology of MRSA infections in the Veterans Affairs Healthcare System from 2002 through 2009. ${ }^{5}$ This study demonstrated that the incidence of MRSA infections increased in all healthcare settings in the Veterans Affairs (VA) system, including hospitalized patients, patients in long-term care, and outpatients. The most common sites of infection involved the skin and soft tissue. Pneumonia occurred in $16 \%$ of the hospitalized patients and in $2 \%$ of the outpatients. Mortality rates were $6 \%$ for inpatients and $23 \%$ for patients in long-term care units. There was no information on mortality for outpatients since presumably patients this sick would be admitted to the hospital. The exact site of infection was unclear in approximately $50 \%$ of the outpatients in this study. Consequently, the frequency of some clinical syndromes associated with outpatient MRSA infections in this study is uncertain, and some types of infection are probably under reported. As expected, patients in this Veterans Affairs Healthcare System had significant co-morbidity. Twenty percent or more of the inpatient population had chronic renal disease, chronic respiratory disease, congestive heart failure, depression, diabetes, hypertension, and/or peripheral vascular disease. Important co-morbidity in the outpatient clinics included chronic respiratory disease (23\%), depression (32\%), diabetes (34\%), and hypertension (62\%).

The Active Bacterial Core Surveillance 
MRSA from the Centers for Disease Control and Prevention (CDCP) project provided national estimates of invasive MRSA infections and deaths. These Active Bacterial Core Surveillance reports classify MRSA infections into three categories. ${ }^{6,7}$ Hospital-onset MRSA infections are defined as infections occurring more than two days after hospital admission. Health-care associated MRSA infections are defined as infections occurring $\leq 2$ days after admission and having one or more of the following: i) a history of hospitalization, surgery, dialysis or residence in a long term care facility in the previous year, or ii) the presence of a central vascular catheter. Community-associated (acquired) MRSA infections meet none of the criteria for hospital-onset or health-care associated infections. In 2008, the Active Bacterial Core Surveillance MRSA study provided national estimates of invasive MRSA infections and deaths (29.5 infections per 100,000 and 5.0 deaths per100,000). Approximately $17 \%$ of the infections were community-acquired. CA-MRSA frequently infects a younger population (median age 30 ) and African-Americans (60.1 per 100,000 African Americans vs. 24.5 per 100,000 Caucasians). ${ }^{6}$ While certain clinical presentations are more frequent in CA-MRSA infections than HAMRSA infections, there are no unique associations, and Figure 1 illustrates the percent of cases associated with particular clinical presentations in 2008 collected by the CDCP Active Bacterial Core Surveillance program. ${ }^{6}$ Most MRSA infections reported in this study presented with bacteremia (approximately $70 \%$ in CA-MRSA infections and $>95 \%$ in HAMRSA infections). Pneumonia occurs in 16\% of patients with MRSA infections. The mortality rate in CA-MRSA cases was $9.8 \%$; the mortality rates in hospital-onset and hospital-associated infections were $23.9 \%$ and $14.9 \%$, respectively. In summary, CA-MRSA infections often occur in younger, healthier persons and have a significant mortality rate, but this depends on the clinical setting. These CDCP reports provide information on selected populations with invasive disease. Therefore, the Veterans Affairs and the CDCP studies do not provide a comprehensive description of the epidemiology of the MRSA infections in the United States but do provide information relevant to hospitalized patients. The results in MRSA investigations will depend on case definition, population type, disease activity (endemic vs. epidemic), and completeness of microbiological investigations used to characterize the bacterial isolates.

Staphylococcal pneumonia usually develops after viral infections of the lower respiratory tract which allow staphylococcal adherence to epithelial surfaces and invasion. ${ }^{3}$ Gillet and coworker reported studies on 16 patients with necrotizing pneumonia secondary to
Staphylococcus aureus strains producing the Panton-Valentine leukocidin (PVL). ${ }^{1}$ These patients were young with a median age of 15 and healthy. Seventy-five percent had an influenza-like syndrome prior to presentation. They presented with high temperatures, tachycardia, tachypnea, cyanosis, hypotension, and hemoptysis (38\%). The median trough white blood cell was $1.8 \mathrm{kU} / \mathrm{L}$, and the median trough platelet count was $70 \mathrm{kU} / \mathrm{L}$. Most (86\%) required mechanical ventilation, and the mortality rate was $75 \%$ even though none had an underlying disorder. Most of these bacterial isolates were methicillin-sensitive. Autopsies revealed massive ulceration of the tracheobronchial tree with adherent staphylococci and alveolar hemorrhage and necrosis with large clusters of staphylococci. Vardakas and colleagues recently analyzed case reports with PVL positive community-acquired pneumonia in patients with methicillin-resistant Staphylococcus aureus (MRSA) and determined that these patients often have influenza-like symptoms, complicated hospital courses with acute respiratory failure and multiorgan system failure, and high mortality (50\%). ${ }^{8}$

Community-acquired methicillin-resistant Staphylococcus aureus (CA-MRSA) infections frequently involve the skin and soft tissues and then disseminate to visceral organs such as lung and bone. Swaminathan and colleagues reported a case with staphylococcal skin abscesses complicated by septic arthritis, multiple metastatic bone and soft tissue infections, and cavitary pneumonia secondary to a community-acquired methicillin-sensitive Staphylococcus aureus infection which was
PVL positive. ${ }^{9}$ These authors suggested that their patient had the $P V L$ syndrome characterized by necrotizing pneumonia, severe soft tissue and bone infection, and deep vein thrombosis (DVT). These patients are typically young and healthy and may or may not have a history of skin infection. The DVT presumably develops as a consequence of venous injury related to the adjacent cellulitis. Vardakas' literature review reported that DVT is relatively frequent in patients with both MRSA and methicillin-sensitive Staphylococcus aureus (MSSA) infections even without cellulitis and has a strong association with mortality. ${ }^{8}$ Radiological studies have demonstrated that patients with MRSA pneumonia more frequently have peripheral infiltrates and bilateral pleural effusions than patients with MSSA pneumonia. ${ }^{10}$ Patients with CA-MRSA pneumonia can have either segmental and non-segmental consolidation and/or nodules, and cavities can occur both in areas of consolidation and in nodules. ${ }^{11}$ These patterns probably reflect different routes of lung infection and highlight the potential for necrosis in these infections.

\section{How are genetic studies used to track the epidemiology of staphylococcal infections?}

Multilocus sequence typing (MLST) is the preferred sequencing method to study the molecular evolution of Staphylococcus aureus. ${ }^{12-16}$ By sequencing seven housekeeping gene loci ( $\operatorname{arcC}$, aroE, glpF, gmk, pta, tpi and yqiL), each strain of Staphylococcus aureus is

\section{Clinical Presentations of MRSA Infections}

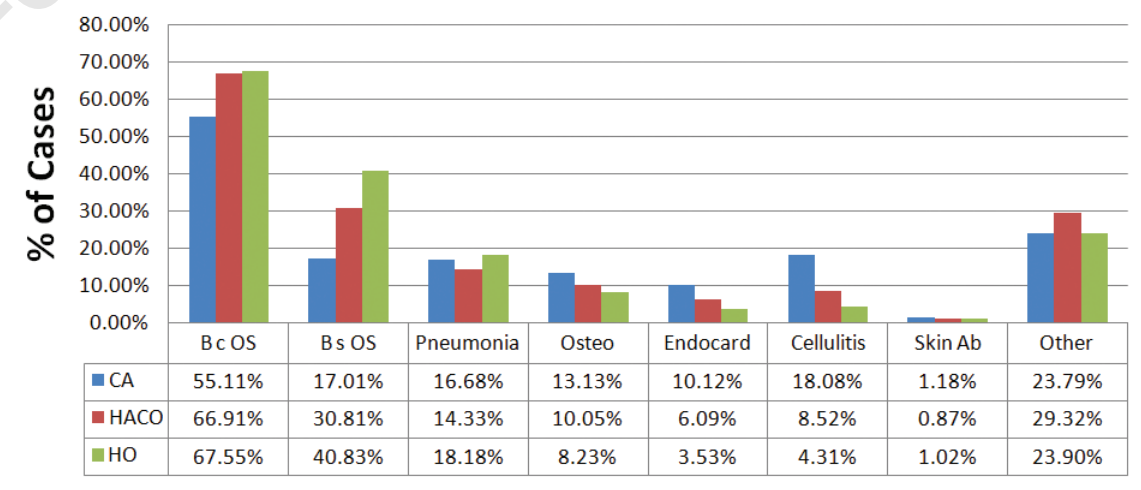

Figure 1. Values represent the percentage of a given clinical presentation of the total number of reported cases for a particular clinical classification. The percentages may exceed $100 \%$ because one infection may have several areas of clinical involvement. This information is based on the Centers for Disease Control and Prevention Active Bacterial Core Surveillance program (2008), and these results represent 929 cases with communityacquired Methicillin-resistant Staphylococcus aureus, 3203 cases with hospital-acquired Methicillin-resistant Staphylococcus aureus, and 1276 cases with hospital-onsetMethicillin-resistant Staphylococcus aureus. CA, community-acquired; HACO, health care-associated community onset; $\mathrm{HO}$, hospital onset; $\mathrm{BcOS}$, bacteremia with other syndromes; BsOS, bacteremia only; Osteo, osteomyelitis; Endocard, endocarditis; Skin Ab, skin abscesses, including skin abscesses, necrotizing fasciitis, gangrene, wounds. 
assigned an allelic profile consisting of seven integers. ${ }^{15}$ Each seven integer allelic profile defines a sequence type. Sequence types that match in $\geq 5$ allelic positions are designated as a clonal complex, a group of Staphylococcus aureus that likely evolved from a single source. ${ }^{13}$ There are multiple clonal complexes, each with many sequence types. Pulsed-field gel electrophoresis (PFGE) is also used to investigate HA-MRSA and hospital to hospital transmission. ${ }^{15}$ This method uses digests of the bacterial DNA and gel electrophoresis to create patterns of DNA bands. PFGE differs from conventional electrophoresis because the voltage is sequentially applied to the gel in three different directions to increase the resolution of the bands. This allows researchers to examine large fragments of up to $40 \times 10^{6}$ base pairs, whereas conventional gel electrophoresis allows examination only of up to $50 \times 10^{3}$ base pairs of DNA. ${ }^{16,17}$ The pattern of bands is used to identify (using reference standards) and compare isolates recovered in epidemiological investigations. In 2003, McDougal, et al. identified eight lineages designated as pulsed-field types USA100 through USA800. In order of decreasing prevalence, CA-MRSA infections are usually caused by USA types 300,1100 , 1000 , and 500, and HA-MRSA infections are primarily caused by USA100 and 200.18,19 Staphylococcal Protein A (spa) is another sequence-based method to determine genetic relatedness of Staphylococcal isolates using the variable region of an internal fragment of a sin- gle gene. Studies in Europe using spa typing suggest that there is more geographic clustering in MRSA isolates than MSSA isolates and that that MRSA isolates have less genetic diversity. ${ }^{20}$ Faria and coworkers compared typing methods using both MRSA and MSSA strains and suggested that PFGE and spa typing provide good discrimination (typing) for local epidemiological studies and can suggest clonal relationships and lineages. ${ }^{20}$ Finally, SCCmec typing (see section below on methicillin resistance) is also used to characterize MRSA isolates. Table 1 provides a summary of the genetic characteristics of four important US clones. MLST, PFGE, SCCmec, and spa typing clearly help characterize bacterial isolates causing infection and allow epidemiological tracking. These methods do not provide unequivocal identification of the origin staphylococcal isolates. In addition, the absence of the HA-MRSA criteria used by the CDCP does not consistently predict the presence of common CA-MRSA associations, such as clindamycin/ ciprofloxacin susceptibility, the presence of SCCmec IV or V, the presence of PVL, and a lack of MRSA history. ${ }^{21,22}$ This difficulty in making an accurate diagnosis of either CA- or HAMRSA with current criteria indicates a need for different approaches to classifying these bacteria. This has become even more important since recent MRSA multi-locus sequence typing and pulsed-field gel electrophoresis studies have demonstrated that CA-MRSA can occur in hospital environments. ${ }^{22,23}$
Why are methicillin-resistant Staphylococcus aureus isolates resistant to methicillin?

SCCmec typing by PCR directly identifies methicillin sensitivity or resistance. ${ }^{24,25}$ SCC stands for staphylococcal cassette chromosome, which is a genomic island, also called a mobile genetic element, passed between staphylococcal bacteria by an unknown mechanism(s). Traditionally, the SCCmec element has been thought to consist of a mec complex, cassette chromosomal recombinases (ccr), additional drug resistance genes, and junkyard regions $(\mathrm{J})$. The mec complex carries the mecA gene, whose protein product is a penicillinbinding protein that has no or limited affinity for $\beta$-lactam antibiotics and thus confers $\beta$-lactam resistance.

There are seven different SCCmec types depending on different base sequences of the mec complex and the ccr genes. SCCmec types are clinically relevant since type I, IV, V, VI, and VII only carry resistance to $\beta$-lactam antibiotics. Type IV is typically found in CA-MRSA and represents a key difference from HA-MRSA (Tables 1 and 2). ${ }^{18}$ CA-MRSA is susceptible to non- $\beta$-lactam antibiotics, with some exceptions, while HA-MRSA, typically associated with SCCmec types II and III, usually carry drug resistance genes to tetracyclines, flouroquinolones, macrolides, aminoglycosides, lincosamides, glycopeptides, and streptogramins. $^{26-28}$ Pulsed-field type USA300 (ST8

Table 1. Genetic analysis of Methicillin-resistant Staphylococcus aureus isolates.

\begin{tabular}{|c|c|c|c|c|c|}
\hline Example & PFGE Clone & MLST & SPA & mecA gene & Comment \\
\hline$\# 1$ & USA100 & ST 5 & TJMBMDMGMK & II & Hospital-acquired \\
\hline$\# 2$ & USA200 & ST 36 & WGKAKADMQQQ & II & Hospital-acquired \\
\hline$\# 3$ & USA300 & ST 8 & YHGFMBQBLO & IV & Community-acquired \\
\hline$\# 4$ & USA400 & ST 1 & VJJJFF & IV & Community-acquired \\
\hline
\end{tabular}

PFGE, pulsed-field gel electrophoresis; MLST, multilocus sequence type; SPA, staphylococcal protein A motif (nomenclature for pattern of repeat base pair sequences in the C-terminal region of the protein A gene).

Table 2. Genetic differences between community-acquired Methicillin-resistant Staphylococcus aureus and hospital-acquired Methicillin-resistant Staphylococcus aureus.

\begin{tabular}{|c|c|c|c|c|}
\hline Phenotype/Genotype & Function & Assay & HA-MRSA & CA-MRSA \\
\hline $\begin{array}{l}\text { Staphylococcal Chromosomal Cassette } \\
\text { (SCCmec) }\end{array}$ & $\begin{array}{l}\beta \text { lactam resistance, additional drug resistance, } \\
\text { virulence role (PSMmec) }\end{array}$ & Multiple & II, III & $\mathrm{IV}, \mathrm{V}$ \\
\hline $\begin{array}{l}\text { Panton-Valentine Leukocidin } \\
\text { (PVL) }\end{array}$ & Cytolysis & Multiple & $(-)$ & $(+)$ \\
\hline $\begin{array}{l}\text { Phenol Soluble Modulin } \\
\text { (PSM) }\end{array}$ & Cytolysis, hemolysis, inflammation & Multiple & $(+)$ & $(+)$ \\
\hline $\begin{array}{l}\text { Toxic Staphylococcal Shock Toxin } \\
\text { (TSST-1), } \\
\text { Enterotoxins }\end{array}$ & $\begin{array}{l}\text { Toxic shock syndrome } \\
\text { Gastroenteritis }\end{array}$ & $\begin{array}{l}\text { Some } \\
\text { Some }\end{array}$ & $(+)$ & $(-)$ \\
\hline $\begin{array}{l}\text { Accessory Gene Regulator } \\
\text { (Agr) }\end{array}$ & $\begin{array}{l}\text { Quorum-sensing, determines } \\
\text { adhesion or exotoxin phenotype }\end{array}$ & Some & II, III & I, III (USA 1100 only) \\
\hline
\end{tabular}

HA-MRSA, hospital-acquired Methicillin-resistant Staphylococcus aureus; CA-MRSA, community-acquired Methicillin-resistant Staphylococcus aureus. This table presents an overview of approaches to characterizing MRSA isolates and is not meant to be comprehensive. 
when MLST is used) is responsible for up to 97-99\% of CA-MRSA infections and is typically associated with SCCmec type IV. ${ }^{26}$ Diep and coworkers suggested that the only biological marker that completely differentiates CAMRSA from HA-MRSA is the SCCmec type. According to his 2006 study, all CA-MRSA strains (USA300, 500, 1000, and 1100) carried SCCmec IV. In contrast, all HA-MRSA strains (USA100 and 200) carried SCCmec II. ${ }^{17}$

\section{Do studies on virulence factors explain the pathogenesis of methi- cillin-resistant Staphylococcus \\ aureus infections?}

Despite its broader antibiotic sensitivity, CA-MRSA has pronounced virulence and can cause life-threatening infections, such as necrotizing fasciitis, necrotizing pneumonia, and severe staphylococcal sepsis. ${ }^{26-30}$ Since these presentations occur less frequently in HA-MRSA infections, CA-MRSA must have either unique virulence factors, different combinations of virulence factors, or overexpression of common staphylococcal virulence factors when compared to HA-MRSA. Diep's analysis of 34 virulence factors in well characterized isolates of MRSA demonstrated that PantonValentine Leukocidin (PVL) was the only factor unique to USA300 and 1100 strains (the most common causes of CA-MRSA infections) and was not present in USA 500 and 1000 strains. ${ }^{31}$ PVL positive clinical isolates have a strong association with severe CA-MRSA infections, and experimental studies support PVL's role in the pathogenesis of pneumonia. 8 ,28,29,31-35 Diep and co-workers have demonstrated that MRSA strain USA300 causes necrosis, edema, and hemorrhage in a rabbit model for pneumonia. ${ }^{36}$ In addition, purified PVL toxin inoculated into the rabbit lung recruits and lyses neutrophils in the lung, resulting in inflammation and mortality. Labandeir-Rey et al. demonstrated that PVL positive Staphylococcus aureus strains caused pneumonia in a murine model and that purified toxin caused lung lesions and a high mortality rate. ${ }^{37}$ The PVL toxin induces pores in neutrophils, increases permeability, and causes cell lysis. Hongo investigated the lytic activities of both phenol soluble modulin $\alpha 3$ (PSM $\alpha 3$ ) and PVL in human and murine models. ${ }^{25}$ Both proteins caused neutrophil lysis, and the rate was significantly greater when both PSM $\alpha 3$ and PVL were present, suggesting a synergistic effect of these two exotoxins. ${ }^{25}$ However, Bubeck Wardenburg and coworkers have reported that PVL does not contribute to the pathogenesis of staphylococcus infection in some strains of mice ${ }^{38,39} \mathrm{CA}$ MRSA strains also have other virulence factors, including $\alpha, \delta$, and $\gamma$ hemolysins. Bubeck Wardenburg demonstrated that alpha hemolysin causes lung injury in a murine model for staphylococcal pneumonia..$^{38,39}$ Consequently, the virulence of CA-MRSA isolates potentially involves several toxins and cannot be consistently attributed to a single toxin, such as PVL.

CA-MRSA's increased virulence could reflect differential exotoxin expression. PVL secretion, along with other staphylococcal exotoxins, is regulated by agr (accessory gene regulator). ${ }^{40}$ The accessory gene regulator is a quorum-sensing control that reacts to bacterial density by changing the transcription rates of two divergent operons controlled by promoters P2 and P3 and has a pivotal role in the expression of staphylococcal exotoxin genes. The accessory gene regulator is classified into four different types (namely, agr I, II, III, and IV). ${ }^{41,42}$ In Diep's study, three agr types were included in his virulence factor panel. CA-MRSA strains almost uniformly have the $a g r$ I genotype; HAMRSA strains have a mixture of $a g r$ II and III genotypes. The agr genes positively regulate the expression of all $S$. aureus hemolysins, PVL, PSM, and the majority of $S$. aureus proteolytic enzymes; this gene regulator likely has a crucial role in CA-MRSA-induced leukopenia and parenchymal necrosis. ${ }^{40,43}$ Strains associated with $\operatorname{agr}$ I have been associated with invasive infections, particularly bacteremia, while strains with agr II and III regulators were more frequently associated with non-invasive infections.

CA-MRSA infections frequently cause skin infections and abscesses. Staphylococcus aureus secretes coagulase and von Willebrand factor-binding protein. ${ }^{44}$ These proteins bind to and activate prothrombin which cleaves fibrinogen to fibrin which in turn promotes abscess formation. Staphylococci replicate in the center of abscesses and potentially enter the circulation following rupture of abscess capsules. Therefore, CA-MRSA virulence depends on the presence of certain toxins and proteins (e.g., PVL, alpha hemolysin, coagulase), the production of these toxins and proteins (controlled by the number of gene copies and the agr regulation), and the presence or absence of antibody to the toxin(s). ${ }^{43}$ The pathogenesis of MRSA infections also involves complex interactions between the bacterial proteins, such as coagulase, and the host defenses. Specific antibodies to some staphylococcal proteins confer protection. ${ }^{44}$

\section{How do clinicians and hospitals identify methicillin-resistant \\ Staphylococcus aureus?}

The initial identification and classification of MRSA infections largely depends on the CDCP Active Bacterial Core Surveillance definitions. Laboratory identification of MRSA typically depends on bacterial cultures which require $20 \mathrm{~h}$ with the use of chromogenic media for reliable negative results. ${ }^{45}$ However, isolation and sensitivity testing, along with confirmation, requires an additional 24 to 48 h. ${ }^{46}$ In cases with possible PVL-positive hypervirulent CA-MRSA infections, this delay in diagnosis could prove fatal. Thus clinicians need real-time identification of MRSA and PVL. MRSA can be identified by the presence of SCCmec, and SCCmec typing can usually distinguish CA from HA-MRSA. However, the primary purpose of early identification of CAMRSA infections is to initiate empiric antibiotic coverage and anticipate complications. Several researchers have developed methods for rapidly identifying a variety of staphylococcal virulence factors, including SCCmec and PVL. ${ }^{47,48}$ Tables 1 and 2 list several of the distinguishing differences between CA and HAMRSA. These methods have not been introduced into routine work in most clinical laboratories.

\section{What are the treatment and pre- vention implications?}

The overall mortality for invasive CA-MRSA infections in the CDCP data collection was $10 \%$.The mortality rate for CA-MRSA necrotizing pneumonia is approximately 50\%, and most patients require mechanical ventilation. The usual diagnostic testing required to identify MRSA depends on current culture methods with unavoidable delays. Therefore, clinicians need to consider the possibility that MRSA is responsible for community-acquired infections and choose antibiotics to cover this pathogen. The Infectious Disease Society of America guideline published in 2011 recommends empiric coverage for MRSA in patients with community-acquired pneumonia if they require ICU admission, have necrosis, or have empyema. This represents an A-III recommendation based on expert opinion. ${ }^{49}$ Vancomycin is the first line treatment and should be used in doses to achieve trough levels of 15-20 $\mu \mathrm{g} / \mathrm{mL}$. However, after a positive culture for MRSA is identified, it is important to determine the vancomycin minimum inhibitory concentration for that particular isolate. Recent studies have demonstrated that Staphylococcus aureus isolates have reduced vancomycin susceptibility based on in vitro laboratory testing; this situation increases the potential for treatment failure, especially in patients with bacteremia. Reduced vancomycin sensitivity is usually defined as a minimal inhibitory concentration greater than $1 \mu \mathrm{g} / \mathrm{mL}$. Mascitti and co-workers reported that approximately $34 \%$ of patients with MRSA infections have reduced vancomycin susceptibility. ${ }^{50}$ This occurred in patients with both methicillin-sensitive Staphylococcus aureus infections and MRSA infections, and multivariate analysis of the data in this study indicated that reduced vancomycin susceptibility was 
associated with prior inpatient vancomycin use, prior inpatient levofloxacin use, and methicillin resistance. If the minimum inhibitory concentration is near the breakpoint for susceptibility $(2 \mu \mathrm{g} / \mathrm{mL})$ or the clinical picture deteriorates, linezolid should be used. Linezolid is a bacteriostatic antibiotic with good penetration into lung tissue and is as least as effective as vancomycin in lung infections. Its side effects include thrombocytopenia, lactic acidosis, and neuropathy, especially with prolonged courses. A recent randomized trial compared linezolid with vancomycin in patients with MRSA nosocomial pneumonia. ${ }^{51}$ This study indicated that the linezolid treated patients had a higher level of clinical success at the end of the study than vancomycin treated patients. The overall frequency of adverse events was similar, but nephrotoxicity occurred more frequently in patients treated with vancomycin (18\%) than with linezolid (8.4\%). Sixty day mortality rates were similar in the two groups. Daptomycin provides an alternative for treating patients with MRSA bloodstream infections and/or endocarditis. However, it is not useful in patients with pneumonia because of poor activity levels in the lung probably related to binding to surfactant in alveolar spaces. Patients with CA-MRSA skin and soft tissue infections can often be treated with trimethoprim-sulfa, doxycycline, and clindamycin, but these drugs are not adequate for the initial therapy of critically ill hospitalized patients. In addition, antimicrobials that suppress exotoxin production and secretion should be considered in cases with severe infection. Clindamycin decreases staphylococcal exotoxin production in vivo and in vitro, and some authors think that the initial therapy should include linezolid and clindamycin.52 However, some staphylococcal isolates have inducible clindamycin resistance which can be detected in the laboratory using the $D$ test. Inducible and constitutive clindamycin resistance occurs more frequently in MRSA isolates. ${ }^{53}$ In very sick patients with multiorgan failure or shock, intravenous immunoglobulin might be considered since it neutralizes PVL in vitro. ${ }^{33,54}$ It has been used in a small number of cases at doses of $2 \mathrm{gm}$ per kilogram with a second dose at 48 h. Molecular diagnostic techniques can now provide relatively rapid identification of methicillin-resistant staphylococci on body surfaces and in clinical specimens (Table 2). Some healthcare systems have introduced screening for nasal colonization by MRSA in patients admitted to various hospital services. These screening protocols range from the selected screening in specialized populations, such as patients with a prior history of MRSA infection, to universal screening of all patients admitted to the hospital. This information can be used to determine which patients need con- tact isolation and can be used for clinical decision making in patients who present with clinical syndromes consistent with staphylococcal infection. In addition, some clinical services make an effort to eradicate nasal colonization with MRSA in patients who are at risk for staphylococcal infection following either surgery or various procedures, such as long term vascular catheterization. The approach to the management of patients with nasal colonization with MRSA must consider the following issues: i) MRSA colonization may resolve spontaneously and then recur; ii) Treatment of MRSA colonization with either oral or local antibiotics may not produce sustained eradication; iii) Given these uncertainties, selective eradication seems to provide the best strategy. For example, treating MRSA colonization in patients on chronic dialysis could potentially reduce the frequency of MRSA related infection. ${ }^{55,56}$ In general, the management of colonized patients will depend on the prevalence of MRSA in the hospital and in select patient populations in that particular hospital, on the resources available for testing and treatment, and on the overall infectious control policies and priorities.

\section{Conclusions}

Many clinicians are not aware of the increased disease severity in hypervirulent MRSA infections. CA-MRSA infections can cause both severe fulminant disease including necrotizing pneumonia and chronic infections with multiple abscesses. Some but not all of these Staphylococcal strains secrete the PVL toxin. However, even if PVL- positive CA-MRSA were suspected, most clinical laboratories do not test for PVL. Better and faster identification methods would have both epidemiological and treatment implications. However, until this testing becomes available and is tested in clinical decision-making, clinicians need to understand the clinical importance of CAMRSA and manage these patients as if shock and multiorgan system failure were inevitable. In some patients with possible MRSA infection the initial empiric therapy should include either vancomycin or linezolid.

\section{References}

1. Gillet Y,Vanhems P, Lina G, et al. Factors predicting mortality in necrotizing community-acquired pneumonia caused by Staphylococcus aureus containing PantonValentine leukocidin. Clin Infect Dis 2007; 45:315-21.

2. Buck JM, Como-Sabetti K, Harriman KH, et al. Community-associated methicillinresistant Staphylococcus aureus, Minnesota, 2000-2003. Emerg Infect Dis 2005;11:1532-38.

3 Martin E, Frandah W, Raj R, Nugent K. A young adult with a fulminant Staphylococcus aureus infection and multiple thrombi. Chest Dis Rep 2011;1:e16.

4. Kuehnert MJ, Kduszon-Moran D, Hill HA, et al. Prevalence of Staphylococcus aureus nasal colonization in the United States, 2001-2002. J Infect Dis 2009;28:835-9.

5. Caffrey AR, LaPlante KL. Changing epidemiology of methicillin-resistant Staphylococcus aureus in the Veterans Affairs Healthcare System, 2002-2009. Infection 2011 Dec13. [Epub ahead of print]

6. Centers for Diseases Control and Prevention. 2008. Active Bacterial Core Surveillance Report, Emerging Infections Program Network, Methicillin-Resistant Staphylococcus aureus. 2008. Available from: http://www.cdc.gov/abcs/reports-findings/survreports/mrsa08.pdf

7. Centers for Disease Control and Prevention. 2007 Active Bacterial Core Surveillance Report, Emerging Infections Program Network, Methicillin-Resistant Staphylococcus aureus. 2007. Available from: http://www.cdc.gov/abcs/reports-findings/survreports/mrsa07.pdf

8. Vardakas KZ, Matthaiou DK, Falagas ME. Comparison of community-acquired pneumonia due to methicillin-resistant and methicillin-susceptible Staphylococcus aureus producing the Panton-Valentine leukocidin. Int J Tuberc Lung Dis 2009;13: 1476-85.

9. Swaminathan A, Massasso D, GotisGraham I, Gosbell I. Fulminant methicillinsensitive Staphylococcus aureus infection in a healthy adolescent, highlighting 'Panton-Valentine leukocidin syndrome'. Intern Med 2006;36:744-7.

10. Morikawa K, Okada F, Ando Y, et al. Methicillin resistant Staphylococcus aureus and methicillin-sensitive susceptible $\mathrm{S}$ aureus pneumonia: comparison of clinical and thin-section CT finding. Br J Radiol 2011 July 12. [Epub ahead of print]

11. Nguyen ET, Kanne JP, Hoang LM, et al. Community acquired methicillin-resistant Staphylococcus aureus pneumonia: radiographic and computed tomography findings. J Thorac Imaging 2008;23:13-9.

12. Naimi T, LeDell K, Como-Sabetti K, et al. Comparison of community- and health care-associated methicillin resistant Staphylococcus aureus infection. JAMA 2003;290:2976-84.

13. Boyle-Vavra S, Daum RS. Communityacquired methicillin-reistant Staphylococcus aureus: the role of Panton- 
Valentine leukocidin. Lab Invest 2007;87:39.

14. Deurenberg RH, Stobberingh EE. The evolution of Staphylococcus aureus. Infect, Genet Evol 2008;8:747-63.

15. Enright MC. The evolution of a resistant pathogen - the case of MRSA. Curr Opin Pharmacol 2003;3:474-9

16. Herschleb J, Ananiev G, Schwartz DC. Pulsed-field gel elctrophoresis. Nat Protoc 2007;2:677-84.

17. Diep BA, Carleton HA, Chang RF, et al. Roles of 34 virulence genes in the evolution of hospital- and community-associated strains of methicillin-resistant Staphylococcus aureus. J Infect Dis 2006; 193:1495-503.

18. McDougal LK, Steward CD, Killgore GE, et al. Pulsed-field gel electrophoresis typing of oxacillin-resistant Staphylococcus aureus isolates from the United States: establishing a national database. J Clin Microbiol 2003;41:5113-20.

19. Grundmann H, Aanensen DM, van den Wigngaard CC, et al. Geographic distribution of Staphylococcus aureus causing invasive infections in Europe: a molecularepidemiological analysis. PLoS Med 2010;7:e1000215.

20. Faria NA, Carrico JA, Oliveira DC, et al. Analysis of typing methods for epidemiological surveillance of both methicillinresistant and methicillin-susceptible Staphylococcus aureus strains. J Clin Microbiol 2008;46:136-44.

21. David MZ, Glikman D, Crawford SE, et al. What is community-associated methicillin-resistant Staphylococcus aureus? J Infect Dis 2008;19:1235-43.

22. D'Agata EM, Webb GF, Horn MA, et al. Modeling the invasion of communityacquired methicillin-resistant Staphylococcus aureus into hospitals. Clin Infect Dis 2009;48:274-84.

23. Zhang K, McClure JA, Elsayed S, et al. Novel multiplex PCR assay for characterization and concomitant subtyping of staphylococcal cassette chromosome mec types $\mathrm{I}$ to $\mathrm{V}$ in methicillin-resistant Staphylococcus aureus. J Clin Microbiol 2005;43:5026-33.

24. Hidron AI, Low CF, Honig EG, Blombei HM. Emergence of community-acquired methicillin-resistant Staphylococcus aureus strain USA300 as a cause of necrotising community-onset pneumonia. Lancet Infect Dis 2009;9:384-92.

25. Hongo I, Baba T, Oishi K, et al. Phenol-soluble modulin alpha 3 enhances the human neutrophil lysis mediated by PantonValentine leukocidin. $\mathrm{J}$ Infect Dis 2009;200:715-23.

26. Patel M. Community-associated methicillin-resistant Staphylococcus aureus infections epidemiology, recognition and management. Drugs 2009;69:693-716.

27. Miller LG, Perdreau-Remington F, Rieg G, et al. Necrotizing fasciitis caused by community-associated methicillin-resistant Staphylococcus aureus in Los Angeles. N Engl J Med 2005;352:1445-53.

28. Francis JS, Doherty MC, Lopatin U, et al. Severe community-onset pneumonia in healthy adults caused by methicillinresistant Staphylococcus aureus carrying the Panton-Valentine leukocidin genes. Clin Infect Dis 2005;40:100-7.

29. Ramdani-Bouguessa N, Bes M, Meugnier $\mathrm{H}$, et al. Detection of methicillin-resistant Staphylococcus aureus strains resistant to multiple antibiotics and carrying the Panton-Valentine leukocidin genes in an Algiers hospital. Antimicrob Agents Chemother 2006;50:1083-5.

30. Gonzalez BE, Martinez-Aguilar G, Hulten $\mathrm{KG}$, et al. Severe staphylococcal sepsis in adolescents in the era of communityacquired methicillin-resistant Staphylococcus aureus. Pediatrics 2005; 115:642-8.

31. Diep BA, Palazzolo-Ballance A, Tattevin P, et al. Contribution of Panton-Valentine Leukocidin in community-associated methicllin-resistant Staphylococcus aureus pathogenesis. PLoS One 2008;3; e3198.

32. Bauer CC, Apfalter P, Daxboeck F, et al. Prevalence of Panton-Valentine leukocidin genes in methicillin-resistant Staphylococcus aureus isolates phenotypically consistent with community-acquired MRSA, 1999-2007, Vienna General Hospital. Eur J Clin Microbiol Infect Dis 2009;28:909-12.

33. Gillet Y, Issartel B, Vanhems $\mathrm{P}$, et al. Association between Staphylococcus aureus strains carrying gene for PantonValentine leukocidin and highly lethal necrotising pneumonia in young immunocompetent patients. Lancet 2002;359:7539.

34. Hampson FG, Hancock SW, Primhak RA. Disseminated sepsis due to a PantonValentine leukocidin producing strain of community acquired meticillin resistant Staphylococcus aureus and use of intravenous immunoglobulin therapy. Arch Dis Child 2006;91:201.

35. Loughman JA, Fritz SA, Storch GA, Hunstad DA. Virulence gene expression in human community-acquired Staphylo coccus aureus infection. J Infect Dis 2009;199:294-301.

36. Diep BA, Chan L, Tattevin P, et al. Polymorphonuclear leukocytes mediate Staphylococcus aureus Panton-Valentine leukocidin-induced lung inflammation and injury. PNAS 2010;107:5587-92.

37. Labandeira-Rey M, Couzon F, Boisset S, et al. Staphylococcus aureus Panton-
Valentine leukocidin causes necrotizing pneumonia. Science 2007;315:1130-3.

38. Bubeck Wardenburg J, Patel RJ, Schneewind 0. Surface proteins and exotoxins are required for the pathogenesis of Staphylococcus aureus pneumonia. Infect Immun 2007;75:1040-4.

39. Bubeck Wardenburg J, Bae T, Otto M, et al. Poring over pores: $\alpha$-hemolysin and Panton-Valentine leukocidin in Staphylococcus aureus pneumonia. Nat Med 2007;13:1405-6.

40. Pichon C, Felden B. Small RNA genes expressed from Staphylococcua aureus genomic and pathogenicity islands with specific expression among pathogenic strains. Proc Natl Acad Sci USA 2005;102 :14249-54.

41. Jarraud S, Mougel C, Thioulouse J, et al. Relationships between Staphylococcus aureus genetic background, virulence factors, agr groups (alleles), and human disease. Infect Immun 2002;70:631-41.

42. Adhikari RP, Arvidson S, Novick RP. A nonsense mutation in agrA accounts for the defect in agr expression and the avirulence of Staphylococcus aureus 8325-4 traP::kan. Infect Immun 2007;75:4534-40.

43. Diep BA, Otto M. The role of virulence determinants in community-associated MRSA pathogenesis. Trends Microbiol 2008;16 :361-9.

44. Cheng AG, McAdow M, Kim H, et al. Contribution of coagulases towards Staphylococcus aureus disease and protective immunity. PLoS Pathog 2010; 6:e1001036.

45. Brown DF, Edwards DI, Hawkey PM, et al. Guidelines for the laboratory diagnosis and susceptibility testing of methicillinresistant Staphylococcus aureus (MRSA). J Antimicrob Chemother 2005;56:1000-18.

46. Stoakes L, Reyes R, Daniel J, et al. Prospective comparison of a new chromogenic medium, MRSASelect, to CHROMagar MRSA and mannitol-salt medium supplemented with oxacillin or cefoxitin for detection of methicillinresistant Staphylococcus aureus. J Clin Microbiol 2006;44:637-9.

47. Bittar F, Ouchenane Z, Smati F, et al. MALDI-TOF-MS for rapid detection of staphylococcal Panton-Valentine leukocidin. Int J Antimicrob Agents 2009;34:46770.

48. Renwick L, Hardie A, Girvan EK, et al. Detection of methicillin-resistant Staphylococcus aureus and PantonValentine leukocidin directly from clinical samples and the development of a multiplex assay using real-time polymerase chain reaction. Eur J Clin Microbiol Infect Dis 2008;27:791-6.

49. Liu C, Bayer A, Cosgrove SE, et al. Clinical 
practice guidelines by the Infectious Diseases Society of America for the treatment of methicillin-resistant Staphylococcus aureus infections in adults and children: executive summary. Clin Infect Dis 2011;52:285-92.

50. Mascitti KB, Edelstein PH, Fishman NO, et al. Prior vancomycin use is a risk factor for reduced vancomycin susceptibility in methicillin-susceptible but no methicillinresistant Staphylococcus aureus bacteremia. Infect Control Hosp Epidemiol 2012;33:160-6.

51. Wunderink RG, Niederman MS, Kollef MH, et al. Linezolid in methicillin-resistant Staphylococcus aureus nosocomial pneumonia : a randomized, controlled study. Clin Infect Dis 2012;54:621-9.

52. Stevens DL, Ma Y, Salmi DB, et al. Impact of antibiotics on expression of virulenceassociated genes in methicillin-sensitive and methicillin- resistant Staphylococcus aureus. J Infect Dis 2007;195:202-11.

53. Gauduchon V, Cozon G, Vandenesch F, et al. Neutralization of Staphylococcus aureus Panton Valentine leukocidin by intravenous immunoglobulin in vitro. $\mathrm{J}$ Infect Dis 2004;189:346-53.
54. Prabhu K, Rao S, Rao V. Inducible clindamycin resistance in Staphylococcus aureus isolated from clinical samples. $\mathrm{J}$ Lab Physicians 2011;3:25-7.

55. Tacconelli E, Carmeli Y, Aizer A, et al. Mupirocin prophylaxis to prevent Staphylococcus aureus infection in patients undergoing dialysis: a metaanalysis. Clin Infect Dis 2003;12:1629-38.

56. Xu G, Tu W, Xu C. Mupirocin for preventing exit-site infection and peritonitis in patients undergoing peritoneal dialysis. Nephrol Dial Transplant 2010;25:587-92. 